\title{
Clinical Features and Visual Acuity Outcomes in Culture-Positive Endogenous Fungal Endophthalmitis in Southern China
}

\author{
Fang Duan, Yao Yang, Zhaohui Yuan, Yongxin Zheng, Zhixing Cheng, and Xiaofeng Lin \\ Zhongshan Ophthalmic Center, State Key Laboratory of Ophthalmology, Sun Yat-sen University, Guangzhou 510060, China \\ Correspondence should be addressed to Xiaofeng Lin; linxiaof@mail.sysu.edu.cn
}

Received 12 May 2017; Revised 26 June 2017; Accepted 16 July 2017; Published 13 August 2017

Academic Editor: Stephen Charn Beng Teoh

Copyright (C 2017 Fang Duan et al. This is an open access article distributed under the Creative Commons Attribution License, which permits unrestricted use, distribution, and reproduction in any medium, provided the original work is properly cited.

\begin{abstract}
Purpose. To report the causative organisms, management strategies, and visual outcomes of culture-proven endogenous fungal endophthalmitis in a case series from southern China. Methods. We reviewed the microbiological and medical records of patients with culture-positive endogenous fungal endophthalmitis visiting the Zhongshan Ophthalmic Center, Guangzhou, China, between January 1, 2006, and March 31, 2016. Results. The inclusion criteria were met in 32 eyes of 29 patients. Molds were a common causative organism in 15 patients (51.7\%), while yeasts appeared in 14 patients (48.3\%). Initial visual acuity (VA) at the level of finger counting or better was significantly related to a good visual outcome $(P=0.002)$. Molds as a causative agent were significantly associated with worse visual outcome than yeasts $(P=0.020)$. Conclusion. Molds were a common cause of culture-proven fungal endophthalmitis. Endogenous fungal endophthalmitis is generally associated with poor VA outcomes, especially if caused by molds and if the patient's initial VA is too low to permit finger counting.
\end{abstract}

\section{Introduction}

Endogenous endophthalmitis is an ophthalmic emergency that can have severe sight-threatening complications and generally accounts for $2 \%-18.5 \%$ of all reported endophthalmitis cases [1-6]. Endogenous fungal endophthalmitis is a serious condition with potentially devastating visual outcomes. This condition is usually associated with one or more predisposing systemic conditions, such as diabetes mellitus, liver disease, renal failure, cancer, indwelling catheters, systemic surgery, organ transplantation, acquired immune deficiency syndrome (AIDS), intravenous drug use, and immunosuppressive therapy [7-10]. Occasionally, endogenous fungal endophthalmitis may occur in healthy, immunocompetent patients without any risk factors [11-13].

Fungal infections of endogenous origin can be acquired after systemic infections caused by pathogenic fungi or opportunistic fungi [14]. Many fungi that can cause endogenous fungal endophthalmitis have been reported. Most commonly, endogenous fungal endophthalmitis is associated with Candida or Aspergillus species in the western countries [7-9] and northern China [10]. However, our previous study reported that the mold was predominant in southern China
[6]. The treatments for endogenous fungal endophthalmitis usually include intravitreal antifungal injections, systemic antifungals, and vitrectomy.

The current study aims to report a consecutive series of patients with culture-proven endogenous fungal endophthalmitis treated at a single medical center with specific fungal isolates, treatment strategies, and visual acuity (VA) outcomes.

\section{Materials and Methods}

Microbiological and clinical records were reviewed for all patients undergoing treatment at the Zhongshan Ophthalmic Center, Guangzhou, China, from January 1, 2006, to March 31,2016 , for intraocular culture-proven endogenous fungal endophthalmitis $(n=29)$. This study was performed in compliance with the principles of the Declaration of Helsinki and was approved by the Institutional Ethics Committee of Zhongshan Ophthalmic Center, Sun Yat-sen University.

Intraocular fluid samples were taken from all patients with suspected or diagnosed endogenous fungal endophthalmitis. Fluids from the anterior chamber were aspirated through the limbus using a needle with a $1 \mathrm{~mL}$ syringe. Vitreous specimens were obtained through the pars plana prior to 
intraocular antibiotic injection or vitrectomy. The collected samples were then inoculated onto sheep blood agar and potato glucose agar to be cultured up to 1 week. All the samples were examined daily for detection of fungal growth. Fungi isolates were identified by trained technicians according to the macroscopic characteristics (color and texture) of the colonies. After the list of possible causative organisms had been obtained, the corresponding medical records of patients with positive culture results for fungi were reviewed, and the clinical presentation, treatment strategy, and outcomes were followed and recorded. Treatment and management decisions were made by the ophthalmologist in charge without a predefined study protocol.

The patients were divided into two groups according to whether the final VA reached a favorable visual outcome (at least enough VA to permit finger counting) or not. An independent sample $t$-test, and a chi-square test were used to analyze the difference between the two groups. Fisher's exact test was used if any frequency in the fourfold table was less than five. The level of significance was defined as a $P$ value of less than 0.05 . All the statistical analyses were performed with SPSS software version 16.0.

\section{Results}

A total of 32 eyes from 29 patients with culture-proven endogenous fungal endophthalmitis were identified in a 10 -year period. The average baseline age of these patients was $33.9 \pm 16.2$ years (range from 1 to 60 years). Thirteen patients (44.8\%) were male and $16(55.2 \%)$ were female. The average length of the follow-up period was $3.8 \pm 5.2$ months (range from 1 week to 2 years). A summary of the clinical characteristics of these patients is provided in Table 1. Bilateral involvement was found in 3 cases $(10.3 \%)$, and unilateral involvement was found in 26 cases (89.7\%).

Eleven patients $(37.9 \%)$ had a history of surgery in the past 12 months, among which 5 patients (17.2\%) received percutaneous nephrolithotomy (PNL) and 6 patients $(20.7 \%)$ underwent cesarean section, ankle fracture surgery, hernioplasty, childbirth, induced abortion, and hysterectomy. In addition, ten patients (34.5\%) had no identifiable infection focus responsible for endogenous endophthalmitis.

All 3 cases with bilateral disease were identified to have positive intraocular culture results in at least one eye. The presence of mold (15 patients, 51.7\%) was slightly higher than that of yeasts (14 patients, $48.3 \%$ ). The 15 mold cultures included Aspergillus $(n=7)$, Fusarium $(n=2)$, Mucor $(n=2)$, Helminthosporium $(n=1)$, and Penicillium $(n=1) ; 2$ molds could not be identified. Among the 14 yeast cultures, only 3 were identified as Candida albicans.

Among all 29 patients ( 32 eyes), 26 patients (28 eyes) underwent pars plana vitrectomy (PPV), and 19 patients (21 eyes) were tamponaded with silicone oil subsequently. Three patients could not receive PPV due to severe opacity and edema of the cornea. One patient with bilateral endophthalmitis did not receive surgery in the better eye because the VA had improved after treatment with antifungal medicine.

As shown in Table 2, 30 eyes (27 patients) with initial and final VAs were available for the analysis of possible prognostic factors for the final visual outcome. Two subjects were excluded because they were too young to conduct a VA test. Patients with different levels of initial VA and species of fungi showed significant differences between poor (worse than FC) and relatively good prognosis (FC and better) $(P=0.002$ and $P=0.020$, resp.). Whether the patient underwent surgical treatment and whether the tamponading agent (silicone oil) was used did not affect the final visual outcome $(P=0.563$ and $P=0.225$, resp.).

\section{Discussion}

Endogenous fungal endophthalmitis is a rare intraocular infection resulting from the systemic hematogenous spread of the organism to the eye. In the present study, the medical records and visual outcome of 29 subjects with endogenous endophthalmitis were reviewed. We revealed that a majority of patients had a positive history of surgery, and nearly $1 / 3$ of the cases of endogenous endophthalmitis occurred in otherwise apparently healthy immunocompetent individuals without any infection focus elsewhere in the body. The presence of mold was slightly higher than that of yeasts. The risk factors affecting the visual outcome of endogenous fungal endophthalmitis included initial VA worse than FC and type of fungus (mold).

In previous studies, systemic diseases including diabetes mellitus and renal disease were the main risk factors of endogenous endophthalmitis $[8,15,16]$; however, in our case series, the major predisposing risk factor was recent history of surgery. There were several reasons that may account for this phenomenon. First, the study site is an ophthalmologyspecialized hospital; most patients with severe systemic diseases were more likely to visit general hospitals looking for care from both ophthalmologists and other physicians. Second, the abuse of antibiotics in China [17], especially for postoperative patients, may increase the risk of endogenous fungal infection.

Though in most of the cases of endogenous endophthalmitis one or more risk factors are identified, a few cases among otherwise healthy and immunocompetent individuals have also been reported [11-13]. The etiology and pathogenesis are not fully identified. The visual outcome of such patients is usually poor [13]. The findings in the 10 cases of this study were in agreement with the previous reports. The major organism accounting for these cases was Aspergillus. The mononuclear phagocyte defenses are usually the first line of defense against Aspergillus infections, followed by the neutrophils $[18,19]$. Some deficiency may exist in the mononuclear phagocytes and neutrophils defenses of these patients. However, we did not have records of immunity tests in these patients. A detailed examination of immune function should be conducted for such cases to explore the underlying causes in future work.

Most commonly, yeast was the main cause of endogenous fungal endophthalmitis in western countries [7-9, 20, 21], and most of them were due to Candida. A study in northern China had similar results [10]. However, in our study, the prevalence of mold was more than that of yeast species. Additionally, a lower percentage of Candida was found in 
TABLE 1: Clinical summary of patients with endogenous fungal endophthalmitis.

\begin{tabular}{|c|c|c|c|c|c|c|c|c|c|}
\hline Patients & Age & Gender & Eye & Fungus & Predisposing risk factors & Initial vision & Vitrectomy & Silicone oil & Final vision \\
\hline 1 & 57 & $\mathrm{M}$ & OD & Aspergillus terreus & IT, LD & LP & Yes & Yes & $\mathrm{FC}$ \\
\hline 2 & 2 & $\mathrm{~F}$ & OS & Mold & None & - & Yes & Yes & - \\
\hline 3 & 43 & $\mathrm{~F}$ & OS & Mucor & PNL & $\mathrm{FC}$ & Yes & No & $20 / 63$ \\
\hline 4 & 41 & $\mathrm{~F}$ & OD & Yeast & PNL & FC & Yes & Yes & $20 / 50$ \\
\hline 5 & 60 & M & OD & Yeast & $\begin{array}{l}\text { Cholecystitis } \\
\text { Hernioplasty }\end{array}$ & FC & Yes & No & $20 / 800$ \\
\hline 6 & 35 & $\mathrm{~F}$ & OD & Yeast & Cervicitis & FC & Yes & No & $20 / 800$ \\
\hline 7 & 30 & $\mathrm{~F}$ & OS & Yeast & Hysterectomy & $\mathrm{HM}$ & Yes & Yes & $\mathrm{HM}$ \\
\hline 8 & 28 & M & OU & Yeast & AFS & $\begin{array}{c}20 / 50 \\
20 / 400\end{array}$ & $\begin{array}{l}\text { No } \\
\text { Yes }\end{array}$ & $\begin{array}{l}\text { No } \\
\text { No }\end{array}$ & $\begin{array}{l}20 / 40 \\
20 / 25\end{array}$ \\
\hline 9 & 10 & $\mathrm{~F}$ & OS & Helminthosporium & None & $\mathrm{HM}$ & Yes & Yes & FC \\
\hline 10 & 55 & M & OS & Aspergillus nidulans & $\mathrm{TB}$ & HM & No & No & $\mathrm{HM}$ \\
\hline 11 & 30 & $\mathrm{~F}$ & OS & Aspergillus nidulans & None & 0.1 & Yes & No & NLP \\
\hline 12 & 43 & $\mathrm{~F}$ & OD & Yeast fungus & None & HM & Yes & Yes & LP \\
\hline 13 & 53 & M & OD & Candida albicans & PNL & FC & Yes & Yes & FC \\
\hline 14 & 13 & M & OD & Fusarium & None & LP & No & No & NLP \\
\hline 15 & 6 & M & OD & Aspergillus & None & $20 / 200$ & Yes & Yes & $20 / 400$ \\
\hline 16 & 51 & M & $\mathrm{OU}$ & Candida albicans & PNL & $\begin{array}{l}20 / 200 \\
\text { FC }\end{array}$ & $\begin{array}{l}\text { Yes } \\
\text { Yes }\end{array}$ & $\begin{array}{l}\text { Yes } \\
\text { Yes }\end{array}$ & $\begin{array}{l}20 / 32 \\
20 / 40\end{array}$ \\
\hline 17 & 26 & M & OS & Aspergillus ochraceus & None & LP & Yes & No & $\mathrm{HM}$ \\
\hline 18 & 44 & M & OD & Yeast & Pneumonia & $\mathrm{FC}$ & Yes & Yes & $20 / 800$ \\
\hline 19 & 39 & M & OD & Aspergillus flavus & None & $\mathrm{HM}$ & Yes & Yes & $20 / 400$ \\
\hline 20 & 34 & M & OS & Penicillium & $\mathrm{LD}$ & LP & Yes & No & NLP \\
\hline 21 & 27 & $\mathrm{~F}$ & OD & Yeast & IA & FC & Yes & Yes & $20 / 63$ \\
\hline 22 & 50 & $\mathrm{~F}$ & $\mathrm{OU}$ & Candida albicans & PNL & $\begin{array}{l}\mathrm{FC} \\
\mathrm{HM}\end{array}$ & $\begin{array}{l}\text { Yes } \\
\text { Yes }\end{array}$ & $\begin{array}{l}\text { Yes } \\
\text { Yes }\end{array}$ & $\begin{array}{l}20 / 200 \\
20 / 400\end{array}$ \\
\hline 23 & 26 & $\mathrm{~F}$ & OD & Mucor & $\mathrm{CM}$ & LP & Yes & Yes & HM \\
\hline 24 & 34 & $\mathrm{~F}$ & OS & Yeast & CS & FC & Yes & Yes & $20 / 100$ \\
\hline 25 & 1 & $\mathrm{~F}$ & OS & Fusarium moniliforme & None & - & Yes & Yes & - \\
\hline 26 & 25 & $\mathrm{~F}$ & OS & Yeast & Pneumonia & $\mathrm{FC}$ & Yes & Yes & $20 / 800$ \\
\hline 27 & 25 & $\mathrm{~F}$ & OS & Yeast & Childbirth & $\mathrm{FC}$ & Yes & Yes & $20 / 400$ \\
\hline 28 & 56 & $\mathrm{~F}$ & OS & Mold & None & $\mathrm{HM}$ & Yes & Yes & $\mathrm{HM}$ \\
\hline 29 & 31 & M & OD & Aspergillus flavus & IT & $\mathrm{HM}$ & No & No & $20 / 200$ \\
\hline
\end{tabular}

Note. NLP: no light perception; HM: hand motion; FC: finger counting; OD: right eye; OS: left eye; OU: both eyes; IT: immunosuppressive therapy; LD: liver disease; PNL: percutaneous nephrolithotomy; CS: cesarean section; AFS: ankle fracture surgery; TB: tuberculosis; IA: induced abortion; CM: colpitis mycotica.

our endogenous fungal endophthalmitis cases. Environmental and geographic factors may have contributed to this discrepancy because the spectrum and virulence of causative organisms vary depending on the region and the environment. On the other hand, the patients already had severe endophthalmitis, which is more likely to be associated with mold infection, before they were referred to our institution.

PPV was an important management strategy for patients with endophthalmitis and a surgical method to obtain vitreous samples for diagnosis. An early diagnosis can be made, and interventions including vitrectomy can be carried out to improve the visual outcome [22]. Vitrectomy can reduce the burden of microorganisms and inflammatory mediators present in the vitreous cavity. Lee et al. reported that early vitrectomy in patients with initially good visual presentation was significantly related to a successful visual outcome [23].
In this case series study, $70.8 \%(17 / 24)$ patients who had improved VA outcome underwent simple or combined PPV. This again emphasizes the role of vitrectomy in treating endophthalmitis, even for cases caused by endogenous fungal growth. However, not all patients who underwent PPV showed improved VA after surgery, and chi-square analysis indicated that PPV did not influence the visual outcome significantly. A possible reason may be the late presentation of these patients, implying that early interventions were not made. The small sample size can also produce misleading results. Though PPV may not improve VA in an obvious manner, it is necessary for cases with mass vitreous opacity and rapid progression [24].

Previous studies had found that good initial visual acuity greater than FC was related to a good visual outcome $[23,25]$. This is consistent with our analysis, which suggests that good 
TABle 2: Prognostic factors associated with favorable visual outcome.

\begin{tabular}{|c|c|c|c|}
\hline \multirow[b]{2}{*}{ Factors } & \multicolumn{2}{|c|}{ Final visual outcome } & \multirow[b]{2}{*}{$P$ value } \\
\hline & $\begin{array}{c}\text { FC or better } \\
\text { than FC }\end{array}$ & Worse than FC & \\
\hline \multicolumn{4}{|l|}{ Initial visual acuity } \\
\hline FC or better than FC & 16 & 1 & \multirow{2}{*}{0.002} \\
\hline Worse than FC & 5 & 8 & \\
\hline \multicolumn{4}{|l|}{ Vitrectomy } \\
\hline No & 2 & 2 & \multirow{2}{*}{0.563} \\
\hline Yes & 19 & 7 & \\
\hline \multicolumn{4}{|l|}{ Silicone oil } \\
\hline No & 6 & 5 & \multirow{2}{*}{0.225} \\
\hline Yes & 15 & 4 & \\
\hline \multicolumn{4}{|l|}{ Fungi } \\
\hline Yeast & 15 & 2 & \multirow{2}{*}{0.020} \\
\hline Mold & 6 & 7 & \\
\hline
\end{tabular}

initial VA is an important predictor for visual outcome. Mold endophthalmitis, however, showed worse visual prognosis than yeast cases. This finding agrees with other studies $[10,20]$, and the possible mechanism may be the invasive patterns of Aspergillus infection in the retina and choroid [26]. These findings indicate that earlier and more aggressive interventions should be made for those with mold-induced endophthalmitis to maintain visual acuity as much as possible.

The limitations of the study should be mentioned. This study was retrospectively designed and lacked universal criteria for diagnosis and treatments. The youngest subjects in our series were 1 and 2 years old, limiting the full assessment of visual outcome. Detailed categories of the fungus species were also absent in some cases. Finally, the small sample size may produce bias in the analysis and conclusion.

In conclusion, we assessed the clinical features and visual outcome of 29 patients with culture-proven endogenous fungal endophthalmitis. The major predisposing risk factor was recent history of surgery. The main organism in patients without identifiable systemic risk factors was Aspergillus. The presence of mold was slightly higher than that of yeasts. The factors affecting visual outcome of endogenous fungal endophthalmitis included initial VA and species of fungus. Whether PPV with silicone oil tamponade was used or not may not influence the visual outcome in severe cases.

\section{Conflicts of Interest}

The authors report no conflicts of interest.

\section{Acknowledgments}

This work was supported in part by funds from the National Natural Science Foundation of China (81400381 and 81570839).

\section{References}

[1] C. Bhoomibunchoo, T. Ratanapakorn, S. Sinawat, T. Sanguansak, K. Moontawee, and Y. Yospaiboon, "Infectious endophthalmitis: review of 420 cases," Clinical Ophthalmology, vol. 7, pp. 247-252, 2013.

[2] J. C. Fan, R. L. Niederer, H. von Lany, and P. J. Polkinghorne, "Infectious endophthalmitis: clinical features, management and visual outcomes," Clinical \& Experimental Ophthalmology, vol. 36, no. 7, pp. 631-636, 2008.

[3] C. A. Puliafito, A. S. Baker, J. Haaf, and C. S. Foster, "Infectious endophthalmitis. Review of 36 cases," Ophthalmology, vol. 89, no. 8, pp. 921-929, 1982.

[4] S. K. Shrader, J. D. Band, C. B. Lauter, and P. Murphy, "The clinical spectrum of endophthalmitis: incidence, predisposing factors, and features influencing outcome," The Journal of Infectious Diseases, vol. 162, no. 1, pp. 115-120, 1990.

[5] M. S. Kresloff, A. A. Castellarin, and M. A. Zarbin, "Endophthalmitis," Survey of Ophthalmology, vol. 43, no. 3, pp. 193224, 1998.

[6] F. Duan, K. Wu, J. Liao et al., "Causative microorganisms of infectious endophthalmitis: a 5-year retrospective study," Journal of Ophthalmology, vol. 2016, Article ID 6764192, 7 pages, 2016.

[7] P. P. Connell, E. C. O'Neill, D. Fabinyi et al., "Endogenous endophthalmitis: 10-year experience at a tertiary referral centre," Eye, vol. 25, no. 1, pp. 66-72, 2011.

[8] I. Leibovitch, T. Lai, G. Raymond, R. Zadeh, F. Nathan, and D. Selva, "Endogenous endophthalmitis: a 13-year review at a tertiary hospital in South Australia," Scandinavian Journal of Infectious Diseases, vol. 37, no. 3, pp. 184-189, 2005.

[9] V. Schiedler, I. U. Scott, H. W. Flynn Jr., J. L. Davis, M. S. Benz, and D. Miller, "Culture-proven endogenous endophthalmitis: clinical features and visual acuity outcomes," American Journal of Ophthalmology, vol. 137, no. 4, pp. 725-731, 2004.

[10] H. Zhang and Z. Liu, "Endogenous endophthalmitis: a 10 -year review of culture-positive cases in northern China," Ocular Immunology and Inflammation, vol. 18, no. 2, pp. 133138, 2010.

[11] D. A. Kostick, R. E. Foster, C. Y. Lowder, S. M. Meyers, and M. C. McHenry, "Endogenous endophthalmitis caused by Candida albicans in a healthy woman," American Journal of Ophthalmology, vol. 113, no. 5, pp. 593-595, 1992.

[12] S. Valluri, R. S. Moorthy, P. E. Liggett, and N. A. Rao, "Endogenous Aspergillus endophthalmitis in an immunocompetent individual," International Ophthalmology, vol. 17, no. 3, pp. 131-135, 1993.

[13] K. Shankar, L. Gyanendra, S. Hari, and S. D. Narayan, "Culture proven endogenous bacterial endophthalmitis in apparently healthy individuals," Ocular Immunology and Inflammation, vol. 17, no. 6, pp. 396-399, 2009.

[14] R. C. Vilela, L. Vilela, P. Vilela et al., "Etiological agents of fungal endophthalmitis: diagnosis and management," International Ophthalmology, vol. 34, no. 3, pp. 707-721, 2014.

[15] D. Ratra, K. Saurabh, D. Das et al., "Endogenous endophthalmitis: a 10-year retrospective study at a tertiary hospital in South India," Asia-Pacific Journal of Ophthalmology (Philadelpia, PA), vol. 4, no. 5, pp. 286-292, 2015.

[16] M. I. Binder, J. Chua, P. K. Kaiser, G. W. Procop, and C. M. Isada, "Endogenous endophthalmitis: an 18-year review of 
culture-positive cases at a tertiary care center," Medicine (Baltimore), vol. 82, no. 2, pp. 97-105, 2003.

[17] J. Currie, W. Lin, and J. Meng, "Addressing antibiotic abuse in China: an experimental audit study," Journal of Development Economics, vol. 110, pp. 39-51, 2014.

[18] A. Schaffner, H. Douglas, and A. Braude, "Selective protection against conidia by mononuclear and against mycelia by polymorphonuclear phagocytes in resistance to Aspergillus. Observations on these two lines of defense in vivo and in vitro with human and mouse phagocytes," The Journal of Clinical Investigation, vol. 69, no. 3, pp. 617-631, 1982.

[19] R. D. Diamond, "Inhibition of monocyte-mediated damage to fungal hyphae by steroid hormones," The Journal of Infectious Diseases, vol. 147, no. 1, p. 160, 1983.

[20] J. Sridhar, H. W. Flynn Jr., A. E. Kuriyan, D. Miller, and T. Albini, "Endogenous fungal endophthalmitis: risk factors, clinical features, and treatment outcomes in mold and yeast infections," Journal of Ophthalmic Inflammation and Infection, vol. 3, no. 1, p. 60, 2013.

[21] A. Lingappan, C. C. Wykoff, T. A. Albini et al., "Endogenous fungal endophthalmitis: causative organisms, management strategies, and visual acuity outcomes," American Journal of Ophthalmology, vol. 153, no. 1, article e161, pp. 162-166, 2012.

[22] D. Pittet, M. Monod, P. M. Suter, E. Frenk, and R. Auckenthaler, "Candida colonization and subsequent infections in critically ill surgical patients," Annals of Surgery, vol. 220, no. 6, pp. 751-758, 1994.

[23] S. Lee, T. Um, S. G. Joe et al., "Changes in the clinical features and prognostic factors of endogenous endophthalmitis: fifteen years of clinical experience in Korea," Retina, vol. 32, no. 5, pp. 977-984, 2012.

[24] X. Shen and G. Xu, "Vitrectomy for endogenous fungal endophthalmitis," Ocular Immunology and Inflammation, vol. 17, no. 3, pp. 148-152, 2009.

[25] H. W. Lim, J. W. Shin, H. Y. Cho et al., "Endogenous endophthalmitis in the Korean population: a six-year retrospective study," Retina, vol. 34, no. 3, pp. 592-602, 2014.

[26] C. F. Romero, M. K. Rai, C. Y. Lowder, and K. A. Adal, "Endogenous endophthalmitis: case report and brief review," American Family Physician, vol. 60, no. 2, pp. 510-514, 1999. 


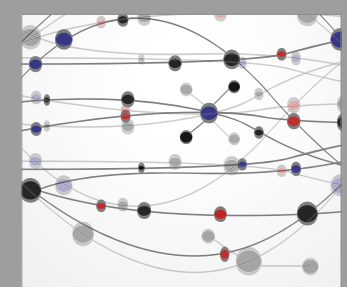

The Scientific World Journal
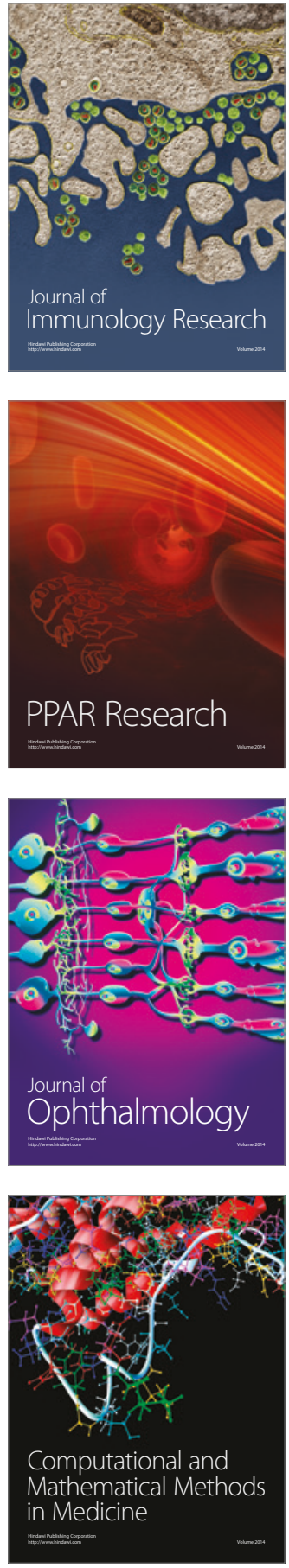

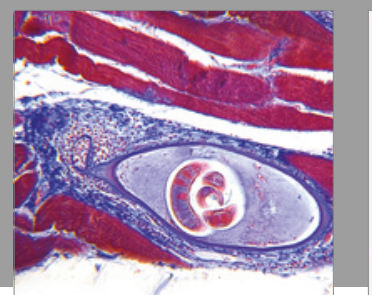

Gastroenterology Research and Practice
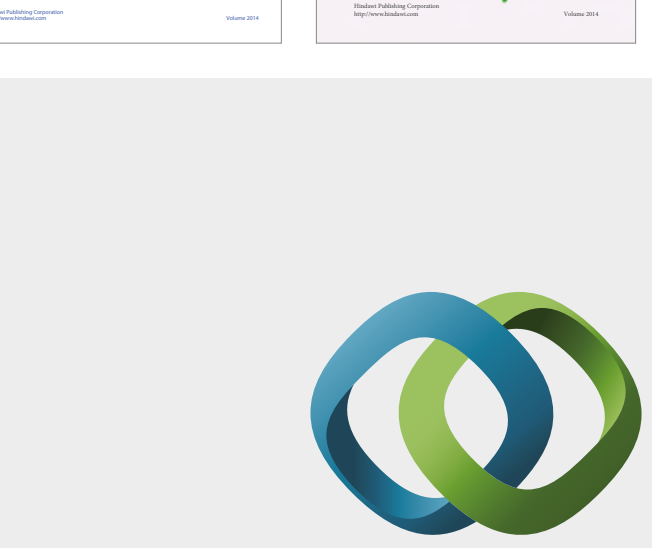

\section{Hindawi}

Submit your manuscripts at

https://www.hindawi.com
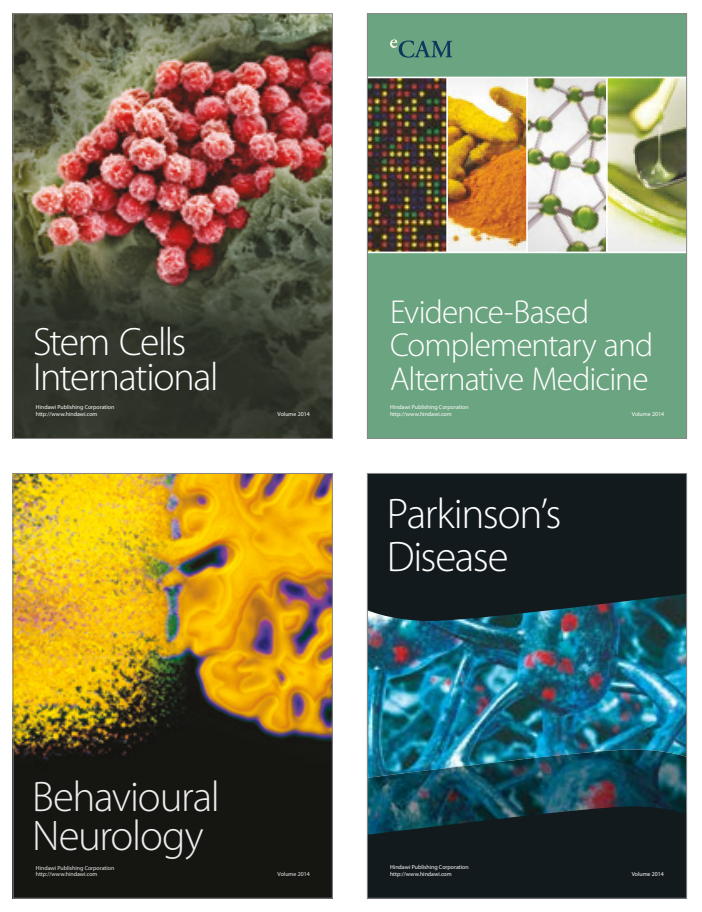
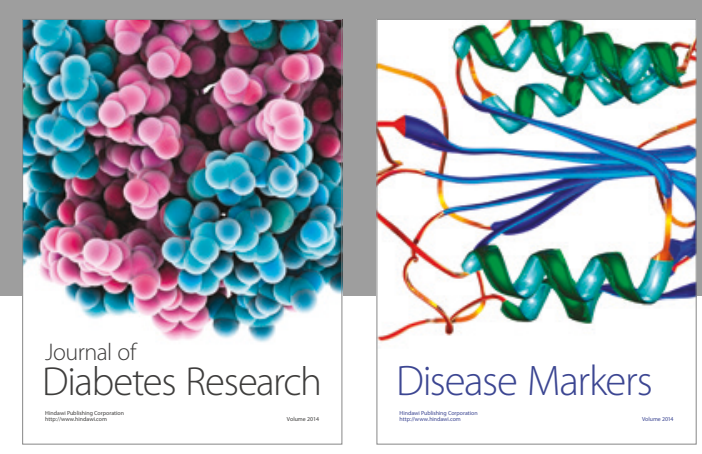

Disease Markers
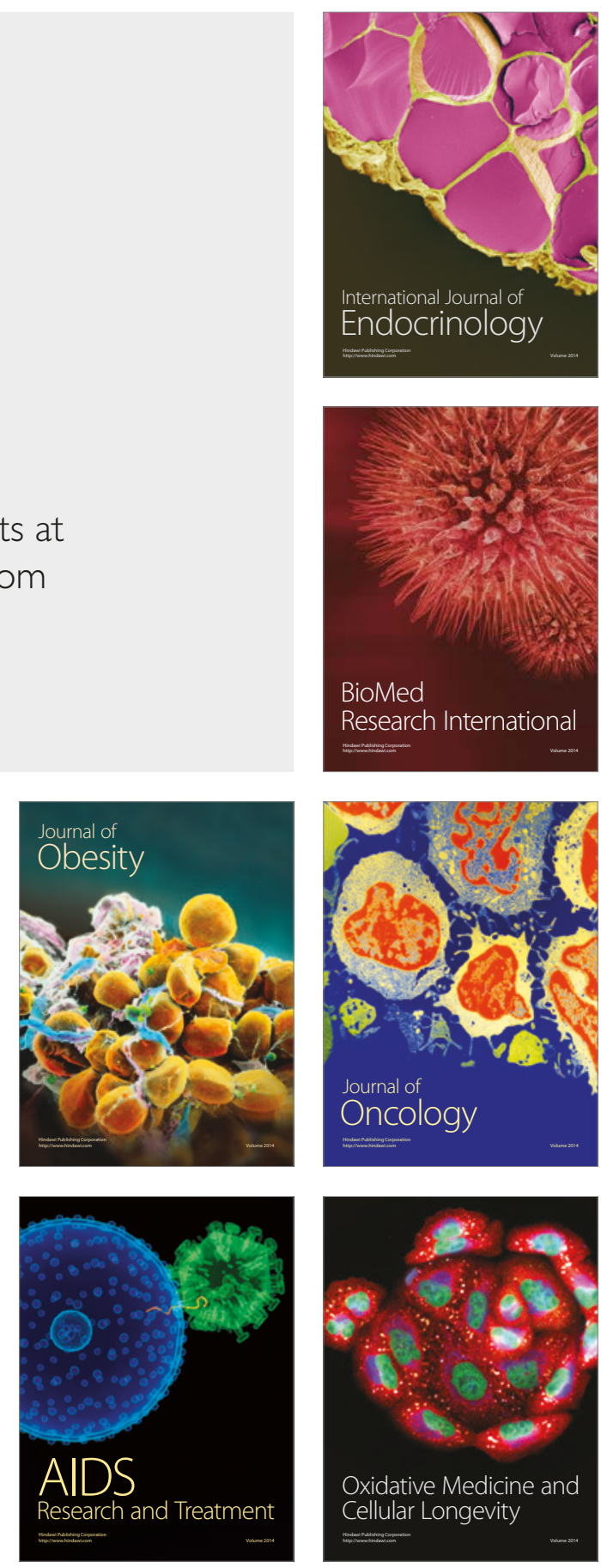